\title{
Electrophysiological and neuropsychological predictors of conversion to schizophrenia in at-risk subjects
}

\author{
Tomiki Sumiyoshi $^{1}{ }^{*}$, Tomohiro Miyanishi $^{2}$, Tomonori Seo $^{2}$ and Yuko Higuchi ${ }^{2}$ \\ ${ }^{1}$ National Center of Neurology and Psychiatry, Kodaira, Tokyo, Japan \\ 2 Department of Neuropsychiatry, University of Toyama Graduate School of Medicine and Pharmaceutical Sciences, Toyama, Japan
}

\author{
Edited by: \\ Ales Stuchlik, Institute of Physiology \\ Academy of Sciences of the Czech \\ Republic, Czech Republic \\ Reviewed by: \\ Martin Lepage, Douglas Mental \\ Health University Institute, Canada \\ Armida Mucci, University of Naples \\ SUN, Italy \\ Tomas Palenicek, Prague Psychiatric \\ Center, Czech Republic \\ *Correspondence: \\ Tomiki Sumiyoshi, National Center of \\ Neurology and Psychiatry, 4-1-1 \\ Ogawa-Higashi, Kodaira, Tokyo \\ 187-8551, Japan \\ e-mail: tomikisumiyoshi840@ \\ hotmail.com
}

\begin{abstract}
Patients with schizophrenia show neurophysiological and psychological disturbances before the onset of the illness. Mismatch negativity (MMN), an event-related potential, has been shown to be associated with cognitive function. Specifically, duration MMN (dMMN) amplitudes have been indicated to predict progression to overt schizophrenia in subjects with at-risk mental state. The aim of this article is to provide a hypothesis that a combined assessment of dMMN and neuropsychological performance would enhance accuracy for predicting conversion to schizophrenia in at-risk subjects. Data from these neurocognitive modalities in subjects with first-episode schizophrenia (FES) are also presented. There is accumulated evidence that converters to schizophrenia among at-risk subjects show significantly smaller dMMN amplitudes than those in healthy control $(\mathrm{HC})$ subjects at the frontal lead before the onset. In fact, the amplitudes in these converters have been reported to be similar to those in FES to begin with. dMMN current source density, by means of low-resolution brain electromagnetic tomography, was significantly lower in FES than $\mathrm{HC}$ subjects, especially in some medial temporal regions which are implicated in the pathophysiology of schizophrenia. Importantly, dMMN current density in the frontal lobe was positively correlated with working memory performance in FES subjects. These findings indicate the utility of the combination of electrophysiological/neuropsychological assessments for early intervention into patients with schizophrenia and high-risk people.
\end{abstract}

Keywords: event-related potentials, mismatch negativity, dMMN, schizophrenia, cognition, early intervention

\section{INTRODUCTION}

Shorter duration of untreated psychosis (DUP) has been associated with better prognosis in schizophrenia (Jackson and McGorry, 2009). Also, early intervention into individuals who are at risk of developing psychosis is important to attain better longterm outcome (Jackson and McGorry, 2009). There is a suggestion that brain-related markers, such as subtle morphological changes revealed by magnetic resonance imaging, may provide a tool to identify at-risk people vulnerable to schizophrenia (Takahashi et al., 2009). Accordingly, we reported the utility of electrophysiological measures, such as event-related potentials (ERPs), as a sensitive and feasible biomarker for the detection of individuals who later developed schizophrenia (Higuchi et al., 2013b) and early intervention into the illness (Higuchi et al., 2013a).

In this paper, we provide a theory for electrophysiological and neuropsychological predictors of outcome in early psychosis. The topics include: (1) cognitive function in prodromal phase psychosis, as measured by neuropsychological performance; (2) the role for mismatch negativity (MMN), a component of ERPs, in early detection of schizophrenia; and (3) three-dimensional current source imaging of MMN and its relation with cognitive performance in early schizophrenia.

\section{THE PSYCHOSIS HIGH-RISK STATE}

The concept of the psychosis high-risk state has been reported in several ways (e.g., Fusar-Poli et al., 2013). Starting treatment in the early phase of psychosis, or minimizing DUP, is important to improve long-term outcome for patients. If we can start intervention in the prodromal phase, it may prevent progression to psychosis. For this purpose, there have been efforts to establish biological or neuropsychological markers to identify high-risk people who are likely to develop schizophrenia later, which is the main focus of this article.

\section{COGNITIVE FUNCTION BASED ON NEUROPSYCHOLOGICAL MEASURES}

There is abundant evidence that cognitive function is impaired in patients with schizophrenia. Usually, the deficit is measured by neuropsychological test batteries, such as the MATRICS Comprehensive Cognitive Battery (Green et al., 2004; Nuechterlein and Green, 2006). In schizophrenia and related psychoses, several domains of cognitive function are disturbed with a 12 standard deviation decline. The cognitive deficit has been reported to provide a vulnerability marker of schizophrenia, so one would expect similar disturbances in high-risk people for the disease. In fact, a recent meta-analysis of cognitive functioning in people at risk for psychosis indicates impairments in almost all cognitive domains which are typically affected in schizophrenia, i.e., executive function, verbal fluency, attention, visual memory, verbal memory, working memory, and social cognition, with a milder degree (Fusar-Poli et al., 2012). 
The Brief Assessment of Cognition in Schizophrenia (BACS) battery (Keefe et al., 2004) is one of the most frequently used tests to evaluate cognitive impairment of schizophrenia in Japan. It takes only approximately $30 \mathrm{~min}$ to complete, and covers key cognitive domains specifically impaired in schizophrenia (Keefe et al., 2004; Kaneda et al., 2007). We recently investigated performance on the BACS in people with at-risk mental state (ARMS), and compared baseline data between subjects who later developed schizophrenia and those who did not (Higuchi et al., 2013b). As demonstrated in Figure 1, the two groups performed differently in working memory, verbal fluency, and attention. These results are generally consistent with the literature (Fusar-Poli et al., 2012), and indicate impairment of frontal lobe function in vulnerable people plays a role in the progression to schizophrenia (Higuchi et al., 2013b; Miyanishi et al., 2013). Specifically, a recent meta-analytic study (De Herdt et al., 2013) reports worse working memory and visual (learning) memory for converters compared to nonconverters, supporting the above concept based on results from a larger number of subjects.

\section{MISMATCH NEGATIVITY}

As discussed, data from neuropsychological performance may provide some information to identify high-risk individuals who later develop psychosis. However, the sensitivity of neuropsychological evaluation to predict conversion to schizophrenia may be less than that of negative symptoms (Riecher-Rossler et al., 2009). This prompts the search for neurocognitive markers from other modalities, such as ERPs and other electrophysiological paradigms.

MMN is a pre-attentive component of ERPs. When auditory cortex automatically detects a change of stimuli, attention shifting occurs in frontal cortex (Jahshan et al., 2012a,b). This neural process generates MMN. For the measurement of MMN, auditory stimuli were delivered to subjects. Standard and target tones with different durations were randomly presented in the case for duration MMN (dMMN). During the measurement, subjects are requested to pay attention to a silent animation movie and ignore the tones. MMN is obtained by subtracting standard waveforms from target waveforms.

One of the strength of $\mathrm{MMN}$ is the limited number of generators, in contrast to the case for P300, another component of ERPs (Figure 2). The generators for MMN are assumed to be located mainly on superior temporal gyrus and prefrontal cortex. This facilitates functional imaging evaluation. Importantly, MMN amplitudes have been shown to be decreased in schizophrenia with a large effect size (Umbricht and Krljes, 2005). Specifically, dMMN amplitudes have been found to be decreased also in ARMS subjects (e.g., Jahshan et al., 2012a).

Figure 3 demonstrated MMN waveforms at the frontal lead for healthy controls (HCs), ARMS subjects, and first-episode schizophrenia (FES). Converter subjects showed reduction in the amplitudes before the onset, similar to patients with FES. By contrast, MMN amplitudes of non-converters resembled those of HCs (Higuchi et al., 2013b). These results are consistent with some recent reports from other groups of investigators (Bodatsch et al., 2011; Atkinson et al., 2012; Jahshan et al., 2012a; Shaikh et al., 2012). A novel finding in our study was a positive correlation between MMN amplitudes and verbal fluency in ARMS subjects (Higuchi et al., 2013b). This indicates word production during a given time would provide an estimate of an electrophysiological activity which is predictive of progression to overt schizophrenia.

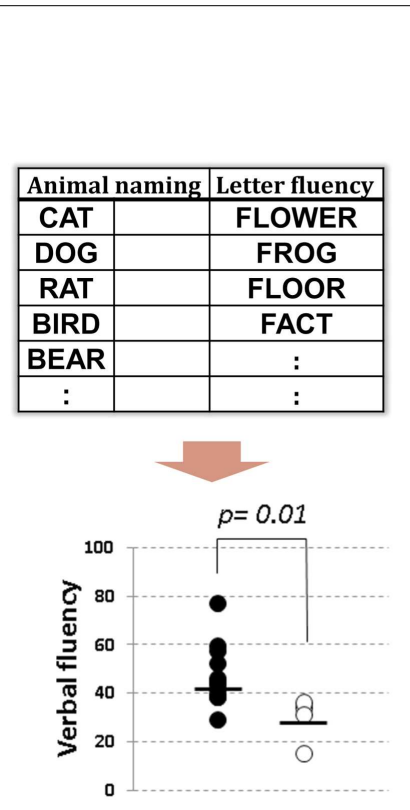

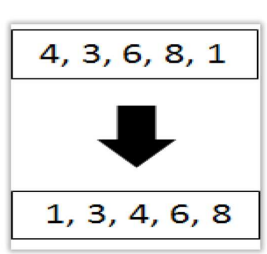

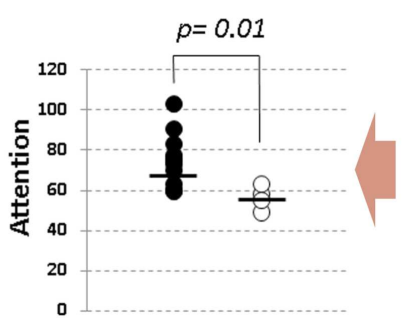

FIGURE 1 | Neuropsychological performance, as evaluated by the BACS, in subjects with at-risk mental state. Transition to overt schizophrenia was predicted by working memory (top), verbal fluency (bottom left), and attention (bottom right) before the onset of illness (extracted from Higuchi et al., 2013 b). 

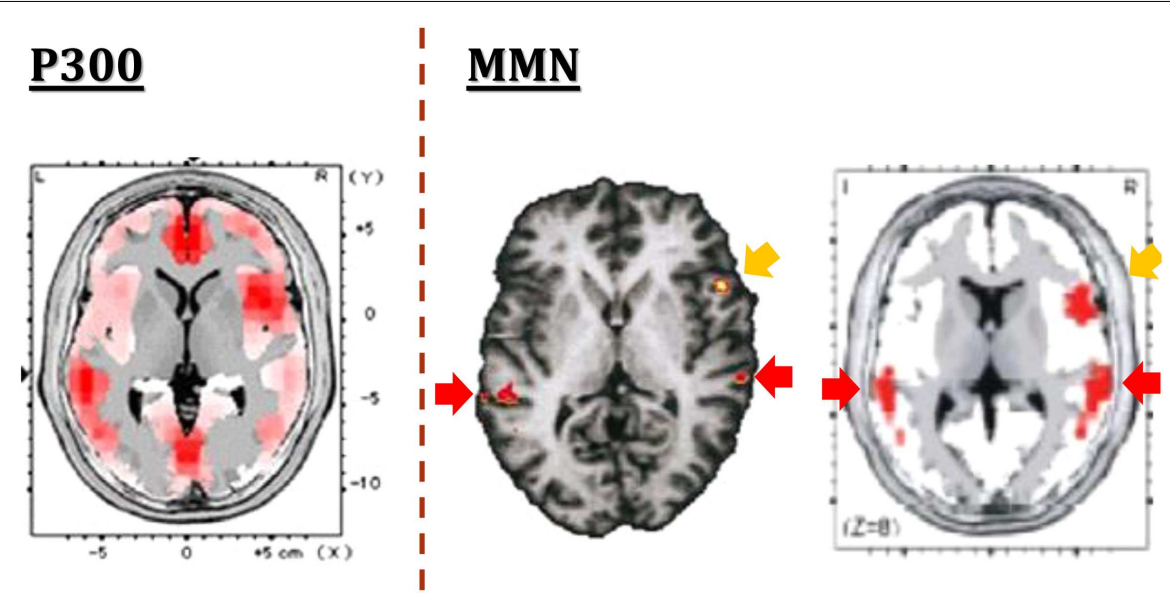

FIGURE 2 |The generators of ERPs. In contrast to multiple generators for P300 (left), putative generators for MMN are limited to superior temporal

EEG-LORETA (right) methods. Images are extracted from Higuchi et al. (2008) gyrus and prefrontal cortex, as demonstrated by fMRI (center) and (left), Opitz et al. (2002) (center), and Marco-Pallares et al. (2005) (right), respectively.
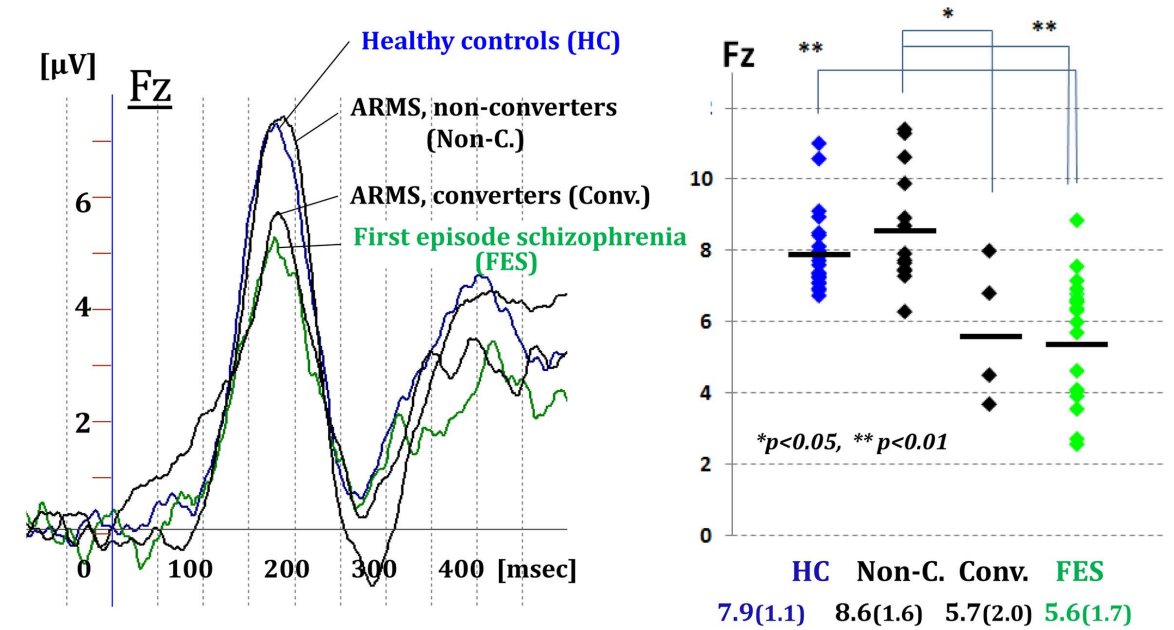

FIGURE 3 | Prediction of transition to schizophrenia by dMMN in ultra-high risk subjects. MMN waveforms at the frontal lead are shown for healthy controls, ARMS subjects, and first-episode schizophrenia. Converter subjects showed reduction in the amplitudes before the onset, similar to patients with first-episode schizophrenia. By contrast, MMN amplitudes of non-converters resembled those of healthy controls (Higuchi et al., 2013b).

\section{OTHER ELECTROPHYSIOLOGICAL AND NEUROPSYCHOLOGICAL BIOMARKERS}

There is evidence that amplitudes of P300, another component of ERPs reflecting attentive cognitive abilities, are reduced in at-risk subjects (e.g., Nagai et al., 2013). As noted above, visual memory has been reported to differentiate between converters and nonconverters in individuals vulnerable to developing schizophrenia (De Herdt et al., 2013). Further efforts are required to refine the use of these biomarkers for early detection of psychosis.

\section{THREE-DIMENSIONAL IMAGING OF dMMN CURRENT DENSITY}

Localization of generators for ERPs provides valuable information. For this purpose, the low-resolution brain electromagnetic tomography (LORETA) methods have been used (Pascual-Marqui, 1999, 2002). In these analyses, current source density of electrical activity is calculated from scalp EEG. Specifically, the LORETA methods can perform voxel-by-voxel comparisons of current source density.

Recently, researchers from the University of California San Diego conducted three-dimensional imaging of dMMN current density in control subjects and patients with chronic schizophrenia (Takahashi et al., 2012). In that study, the mean duration of illness was 24 years, which was lengthy. The comparison between the two groups indicates reduced activations in the cingulate gyrus and medial frontal gyrus in patients (Takahashi et al., 2012).

Regarding the early phase of schizophrenia, we recently reported data from patients whose mean duration of illness was 


\begin{tabular}{lccc}
\hline & $\begin{array}{c}\text { Healthy controls } \\
(\mathbf{n = 2 0})\end{array}$ & $\begin{array}{c}\text { Early schizophrenia } \\
(\mathbf{n = 2 0 )}\end{array}$ & Significance \\
\hline Male/Female & $14 / 6$ & $9 / 11$ & n.s. \\
\hline Age (years) & $25.4(6.9)$ & $27.2(7.3)$ & n.s. \\
Education (years) & $15.1(2.9)$ & $13.2(2.1)$ & $<0.05$ \\
Age at onset (years) & - & $26.5(7.1)$ & \\
Duration of illness (years) & - & $0.6(0.5)$ & \\
\hline
\end{tabular}

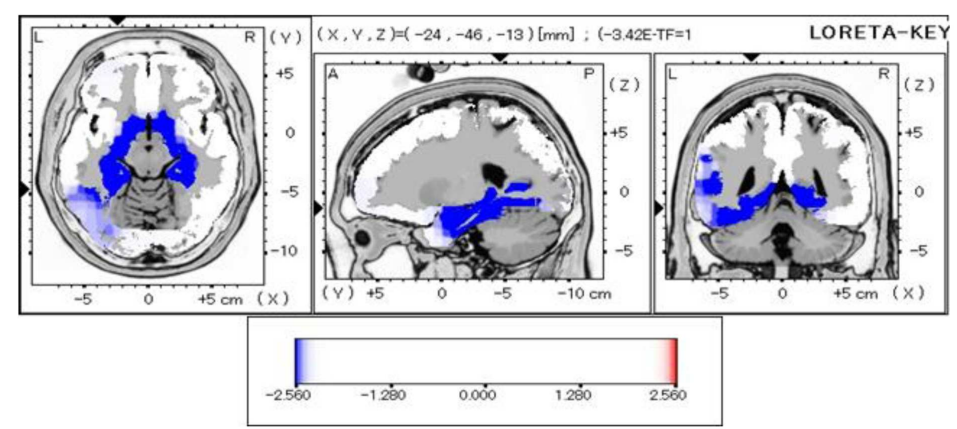

FIGURE 4 | Three-dimensional imaging of dMMN current density in early schizophrenia. Duration of illness was $<1$ year for all patients. Comparison between healthy subjects and patients showed decreased current density in

such brain regions as bilateral parahippocampal gyrus, left fusiform gyrus, right hippocampus, and left anterior cingulate gyrus (data extracted from Miyanishi et al., 2013).

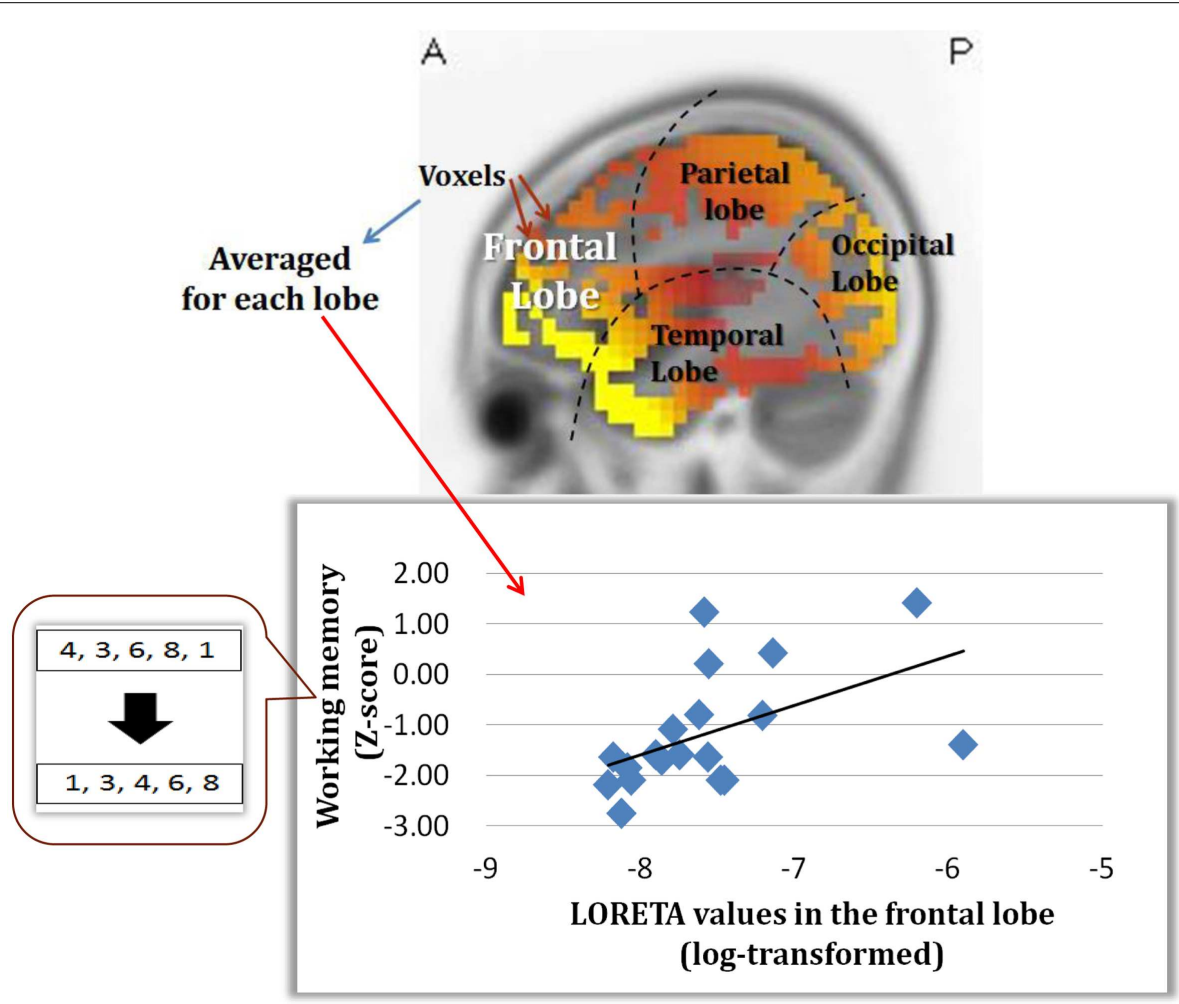

FIGURE 5 | Correlation between dMMN current density in the frontal lobe and working memory, as evaluated by the BACS-J, in patients with early schizophrenia. (Miyanishi et al., 2013). 
$<1$ year (Miyanishi et al., 2013). Figure 4 demonstrates the comparison of dMMN current density between healthy subjects and patients. Early schizophrenia patients showed decreased current density in medial temporal lobe structures and anterior cingulate gyrus, i.e., brain areas related to the pathophysiology of schizophrenia (Jensen et al., 2004; Hao et al., 2009).

An important part of our study was to determine if the change in dMMN activations is associated with neuropsychological performance. As demonstrated in Figure 5, dMMN current density in the frontal lobe is positively correlated with working memory, as measured by the BACS, in early schizophrenia patients (Miyanishi et al., 2013). These findings are consistent with the concept that the prefrontal cortex plays a major role in this cognitive domain. Further study is warranted to see if the association between $\mathrm{dMMN}$ current density in the frontal lobe and working memory is specific to schizophrenia, but not HCs, and if dMMN current density predicts progression to schizophrenia in at-risk people.

\section{REFERENCES}

Atkinson, R. J., Michie, P. T., and Schall, U. (2012). Duration mismatch negativity and P3a in first-episode psychosis and individuals at ultra-high risk of psychosis. Biol. Psychiatry 71, 98-104. doi:10.1016/j.biopsych. 2011.08 .023

Bodatsch, M., Ruhrmann, S., Wagner, M., Muller, R., Schultze-Lutter, F., Frommann, I., et al. (2011). Prediction of psychosis by mismatch negativity. Biol. Psychiatry 69, 959-966. doi:10.1016/j.biopsych. 2010.09.057

De Herdt, A., Wampers, M., Vancampfort, D., De Hert, M., Vanhees, L., Demunter, H., et al. (2013). Neurocognition in clinical high risk young adults who did or did not convert to a first schizophrenic psychosis: a meta-analysis. Schizophr. Res. 149, 48-55. doi:10.1016/j. schres.2013.06.017

Fusar-Poli, P., Borgwardt, S., Bechdolf, A., Addington, J., Riecher-Rossler, A., Schultze-Lutter, F., et al. (2013). The psychosis high-risk state: a comprehensive state-of-the-art review. JAMA Psychiatry 70, 107-120. doi:10.1001/ jamapsychiatry.2013.269

Fusar-Poli, P., Deste, G., Smieskova, R., Barlati, S., Yung, A. R., Howes, O., et al. (2012). Cognitive functioning in prodromal psychosis: a meta-analysis. Arch. Gen. Psychiatry 69, 562-571. doi:10.1001/ archgenpsychiatry.2011.1592

Green, M. F., Nuechterlein, K. H., Gold, J. M., Barch, D. M., Cohen, J., Essock, S., et al. (2004). Approaching a consensus cognitive battery for clinical trials in schizophrenia: the NIMH-MATRICS conference to

\section{CONCLUSION}

This paper provided a hypothesis regarding a role for neuropsychological and electrophysiological markers in intervention into early psychosis and high-risk subjects. The combination of these modalities of neurocognition would be expected to facilitate early detection of subjects who are likely to develop psychosis, and identification of those who need immediate treatment.

\section{ACKNOWLEDGMENTS}

This study was funded by grants-in-aid for Scientific Research from Japan Society for the Promotion of Science, Health, and Labour Sciences Research Grants for Comprehensive Research on Disability, Health, and Welfare (H23-Seishin-Ippan-002; H24Seishin-Ippan-002), and SENSHIN Medical Research Foundation. The authors thank Ms. Shimako Nishiyama, Ms. Yuko Mizukami, Dr. Yasuhiro Kawasaki, Dr. Michio Suzuki, and Dr. Kurachi for support.

illness course of schizophrenia. Psychol. Med. 42, 85-97. doi:10.1017/ S0033291711001061

Jahshan, C., Wynn, J. K., Breitmeyer, B. G., and Green, M. F. (2012b). Nonconscious and conscious color priming in schizophrenia. J. Psychiatr. Res. 46, 1312-1317. doi:10.1016/ j.jpsychires.2012.06.009

Jensen, J. E., Miller, J., Williamson, P. C., Neufeld, R. W., Menon, R. S., Malla, A., et al. (2004). Focal changes in brain energy and phospholipid metabolism in first-episode schizophrenia: 31P-MRS chemical shift imaging study at 4 Tesla. Br. J. Psychiatry 184, 409-415. doi:10.1192/bjp. 184.5.409

Kaneda, Y., Sumiyoshi, T., Keefe, R., Ishimoto, Y., Numata, S., and Ohmori, T. (2007). Brief assessment of cognition in schizophrenia: validation of the Japanese version. Psychiatry Clin. Neurosci. 61, 602-609. doi:10.1111/j. 1440-1819.2007.01725.x

Keefe, R. S., Goldberg, T. E., Harvey, P. D., Gold, J. M., Poe, M. P., and Coughenour, L. (2004). The brief assessment of cognition in schizophrenia: reliability, sensitivity, and comparison with a standard neurocognitive battery. Schizophr. Res. 68, 283-297. doi:10.1016/j.schres. 2003.09.011

Marco-Pallares, J., Grau, C., and Ruffini, G. (2005). Combined ICA-LORETA analysis of mismatch negativity. Neuroimage 25, 471-477. doi:10. 1016/j.neuroimage.2004.11.028

Miyanishi, T., Sumiyoshi, T., Higuchi, Y., Seo, T., and Suzuki, M. (2013). LORETA current source density for duration mismatch negativity and neuropsychological assessment in early schizophrenia. PLOS ONE 8:e61152. doi:10.1371/journal.pone. 0061152

Nagai, T., Tada, M., Kirihara, K., Yahata, N., Hashimoto, R., Araki, T., et al. (2013). Auditory mismatch negativity and $\mathrm{P} 3 \mathrm{a}$ in response to duration and frequency changes in the early stages of psychosis. Schizophr. Res. doi:10.1016/j.schres.2013.08.005

Nuechterlein, K. H., and Green, M. F. (2006). MATRICS Consensus Cognitive Battery. Los Angeles: MATRICS Assessment, Inc.

Opitz, B., Rinne, T., Mecklinger, A., von Cramon, D. Y., and Schroger, E. (2002). Differential contribution of frontal and temporal cortices to auditory change detection fMRI and ERP results. Neuroimage 15, 167-174. doi:10.1006/nimg. 2001.0970

Pascual-Marqui, R. D. (1999). Review of methods for solving the EEG inverse problem. Int. J. Bioelectromagnetism $1,75-86$.

Pascual-Marqui, R. D. (2002). Standardized low-resolution brain electromagnetic tomography (sLORETA): technical details. Methods Find. Exp. Clin. Pharmacol. 24(Suppl. D), 5-12.

Riecher-Rossler, A., Pflueger, M. O., Aston, J., Borgwardt, S. J., Brewer, W. J., Gschwandtner, U., et al. (2009). Efficacy of using cognitive status in predicting psychosis: a 7 year follow-up. Biol. Psychiatry 66, 1023-1030. doi:10.1016/j.biopsych. 2009.07.020

Shaikh, M., Valmaggia, L., Broome, M. R., Dutt, A., Lappin, J., Day, F., et al. (2012). Reduced mismatch negativity predates the onset of psychosis. Schizophr. Res. 134, 42-48. doi:10.1016/j.schres.2011.09.022 
Takahashi, H., Rissling, A. J., Pascual-Marqui, R., Kirihara, K., Pela, M., Sprock, J., et al. (2012). Neural substrates of normal and impaired preattentive sensory discrimination in large cohorts of nonpsychiatric subjects and schizophrenia patients as indexed by $\mathrm{MMN}$ and $\mathrm{P} 3 \mathrm{a}$ change detection responses. Neuroimage $66 \mathrm{C}$, 594-603. doi:10.1016/j.neuroimage. 2012.09.074

Takahashi, T., Wood, S. J., Yung, A. R., Soulsby, B., McGorry, P. D.,
Suzuki, M., et al. (2009). Progressive gray matter reduction of the superior temporal gyrus during transition to psychosis. Arch. Gen. Psychiatry 66, 366-376. doi:10.1001/ archgenpsychiatry.2009.12

Umbricht, D., and Krljes, S. (2005). Mismatch negativity in schizophrenia: a meta-analysis. Schizophr. Res. 76, 1-23. doi:10.1016/j.schres.2004. 12.002

Conflict of Interest Statement: The authors declare that the research was conducted in the absence of any commercial or financial relationships that could be construed as a potential conflict of interest.

Received: 10 July 2013; accepted: 26 September 2013; published online: 21 October 2013.

Citation: Sumiyoshi T, Miyanishi T, Seo $T$ and Higuchi Y (2013) Electrophysiological and neuropsychological predictors of conversion to schizophrenia in at-risk subjects. Front. Behav. Neurosci. 7:148. doi: 10.3389/fnbeh.2013.00148
This article was submitted to the journal Frontiers in Behavioral Neuroscience. Copyright (C) 2013 Sumiyoshi, Miyanishi, Seo and Higuchi. This is an open-access article distributed under the terms of the Creative Commons Attribution License (CC BY). The use, distribution or reproduction in other forums is permitted, provided the original author(s) or licensor are credited and that the original publication in this journal is cited, in accordance with accepted academic practice. No use, distribution or reproduction is permitted which does not comply with these terms. 